\title{
Total endoscopic repair of atrial septal defect under on-pump beating heart
}

\author{
Yihu Tang, Yanhu Wu, Jinfu Zhu, Xiang Liu, Jinxin Zhou, Haobing Huang, Mingke Li, Yawei Dai, Xu Han \\ Department of Cardiovascular Surgery, First Affiliated Hospital with Nanjing Medical University, Nanjing 210029, China \\ Contributions: (I) Conception and design: Y Tang, Y Wu; (II) Administrative support: Y Tang, Y Wu; (III) Provision of study materials or patients: Y \\ Tang, Y Wu; (IV) Collection and assembly of data: Y Tang, Y Wu; (V) Data analysis and interpretation: Y Tang, Y Wu; (VI) Manuscript writing: All \\ authors; (VII) Final approval of manuscript: All authors. \\ Correspondence to: Yanhu Wu. Department of Cardiovascular Surgery, First Affiliated Hospital with Nanjing Medical University, 300 Guangzhou \\ Road, Nanjing 210029, Jiangsu, China. Email: Drwuyanhu@126.com.
}

\begin{abstract}
Background: We previously reported the techniques for total endoscopic atrial septal defect (ASD) repair on hearts arrested with cardioplegia through three small incisions in the chest wall without aid of a surgical robotic system. The optimal results motivated us to use total thoracoscopic technology for ASD on perfused beating hearts.
\end{abstract}

Methods: From 2010 to 2017,161 patients with a mean age of $28.31 \pm 12.34$ years who underwent nonrobotically assisted total thoracoscopic closure for ASD were included in this study, and those patients were also divided into two groups, including group A and group B. In group A, 115 patients underwent the procedure on beating hearts without aorta cross-clamped; in group B, 46 patients underwent the procedure on hearts arrested with cardioplegia with aorta cross-clamped. Cardiopulmonary bypass (CPB) was peripherally achieved as well.

Results: Total thoracoscopic ASD closures were successfully performed without in-hospital mortality or other serious complications in all patients of both groups. Dacron or bovine patches were used in 81 and 32 patients in the two groups, respectively. Duration of operation, duration of CPB, aorta cross-clamped time, duration of mechanical ventilation, the length of intensive care unit (ICU) and post-operative hospital stay in group $\mathrm{A}$, were all shorter than those in group $\mathrm{B}(\mathrm{P}<0.05)$. There was no statistically significant difference in blood transfusion during operation or post-operation thoracic drainage. During follow-up, echocardiograms at 3,30, 90 and 365, showed no residual shunt or tricuspid regurgitation.

Conclusions: Total thoracoscopic closure of ASD without assistance of a surgical robotic system on beating heart is safe and feasible and can be used as a therapeutic option for ASD, and by using the mentioned technique, less injuries are applied to patients.

Keywords: Total thoracoscopy; cardiovascular surgery; atrial septal defect (ASD); congenital heart disease; beating heart

Submitted May 01, 2018. Accepted for publication Oct 11, 2018.

doi: $10.21037 /$ jtd.2018.10.89

View this article at: http://dx.doi.org/10.21037/jtd.2018.10.89

\section{Introduction}

Atrial septal defect (ASD) is the third most common type of congenital heart disease with high risk of development of pulmonary vascular disease (1), accounting for $30-40 \%$ of congenital heart diseases in adults (2). Although catheterization and Amplatzer occlusion devices have been widely applied (3-5), surgical procedures are still required for some large, complex and special ASDs. Over the past few years, a number of endoscopic cardiac procedures with or without the da Vinci Surgical System have been reported (6-8). We previously reported 20 patients who underwent total endoscopic ASD repair on hearts arrested with 
Table 1 Patient baseline characteristics

\begin{tabular}{llll}
\hline Variables & Group A $(\mathrm{n}=115)$ & Group B $(\mathrm{n}=46)$ & P value \\
\hline Age (years) & $27.84 \pm 11.96$ & $29.11 \pm 13.08$ & 0.38 \\
Weight $(\mathrm{kg})$ & $52.13 \pm 12.2$ & $51.93 \pm 8.8$ & 0.93 \\
Female $(\%)$ & $58(50.43)$ & $21(45.65)$ & 0.58 \\
Size of ASD $(\mathrm{mm})$ & $25.96 \pm 6.81$ & $28.64 \pm 8.81$ & 0.07 \\
EF $(\%)$ & $64.53 \pm 8.48$ & $65.61 \pm 4.14$ & 0.51 \\
PASP $(\mathrm{mmHg})$ & $36.47 \pm 11.21$ & $38.68 \pm 13.01$ & 0.63 \\
\hline
\end{tabular}

ASD, atrial septal defect; EF, ejection fraction; PASP, pulmonary arterial systolic pressure.

cardioplegia (9). Based on the previous reports $(6,10-13)$, we improved the techniques and skills of the total endoscopic closure for ASD on beating hearts without the assistance of a robotic surgical system which made the procedure to be much more appropriate and feasible. In the present study, we retrospectively analyzed our clinical experience in total endoscopic ASD repair on beating heart and medical follow-up outcomes.

\section{Methods}

\section{Patient selection}

This study was approved by the Institutional Review Board of the First Affiliated Hospital with Nanjing Medical University (Nanjing, Jiangsu province, China) (No. 2018SR-249). Written informed consent was obtained from the patients for publication of their data and any accompanying images.

From 2010 to 2017, 161 patients underwent total thoracoscopic closure of secundum-type ASD in the Department of Cardiovascular Surgery, First Affiliated Hospital with Nanjing Medical University. These patients were divided into two groups, the first group included patients who underwent closure on beating hearts (group $\mathrm{A}, \mathrm{n}=115$ ), and the second group involved hearts arrested with cardioplegia (group B, $\mathrm{n}=46$ ). The average age of patients was $28.31 \pm 12.34$ years. Of the 161 patients, 79 were female and 82 were male. Preoperative diagnosis, including diameter and anatomic classification of ASD and some other associated anomalies, were investigated through transthoracic two-dimensional (2D) echocardiography, with the preoperative rhythm being sinus rhythm. Patients' baseline characteristics was listed in Table 1. There was no significant difference in the parameters mentioned in Table 1 between the two groups.

The selection criteria for total thoracoscopic ASD repair procedure in our department were listed as following: between 5 and 60 years old; body weight $\geq 15 \mathrm{~kg}$; not appropriate for the interventional closure; pulmonary arterial systolic pressure measured by echocardiograms $\leq 70 \mathrm{mmHg}$; no anomaly of femoral artery and vein; no previous history of surgery or other disease that may cause pleura adhesion on the right chest; without other serious cardiovascular anomalies or chronic illness; ASD patients without valve disease, serious coronary disease, or respiratory disease which may negatively impact pulmonary function.

\section{Surgical repair of ASD}

Our team previously reported the techniques for total thoracoscopic repair of ASD on hearts arrested with cardioplegia (9). After general anesthesia, double-lumen endotracheal tube or bronchial occluder was used to allow for left single-lung ventilation in both groups. Oxygen saturation was continuously monitored as well. Double-lung ventilation was initiated when oxygen saturation fell below $90 \%$. Patients were in the supine position with the right hemithorax slightly elevated to $15-30^{\circ}$, using an inflatable air sack under right subscapular area. A thoracoscope was inserted through a small incision in the chest (Figure 1, Port 1 ) in the fifth intercostal space at the right midaxillary line. The other two incisions were made parasternally in the third intercostal space (Figure 1, Port 2) and in the second intercostal space on the anterior axillary line (Figure 1, Port 3), respectively. Port 2 was used for the entries of surgical instruments and inferior vena cava snare, and Port 3 was used for the entries of the surgical instruments, superior vena cava snare, a cannula for cold perfusion, and an aortic clamp. Soft tissue retractors were used to protect the incisions.

After systemic heparinization, heparin $(3 \mathrm{mg} / \mathrm{kg})$ was used and the activated clotting time (ACT) was more than 420 ms. A catheter (15F, $17 \mathrm{~F}$ or $19 \mathrm{~F}$, Medtronic) was positioned through the right femoral artery into the abdominal aorta. A double-lumen catheter $(24 / 29 \mathrm{~F}$ or $30 / 33 \mathrm{~F}$, Medtronic) was positioned into the inferior and superior vena cava through the right femoral vein to complete the cardiopulmonary bypass (CPB) circuit. The pericardium was opened about $1.5-2 \mathrm{~cm}$ to the phrenic nerve and the incision was extended to visualize the root of aorta and superior vena cava. Pericardium stay sutures were 

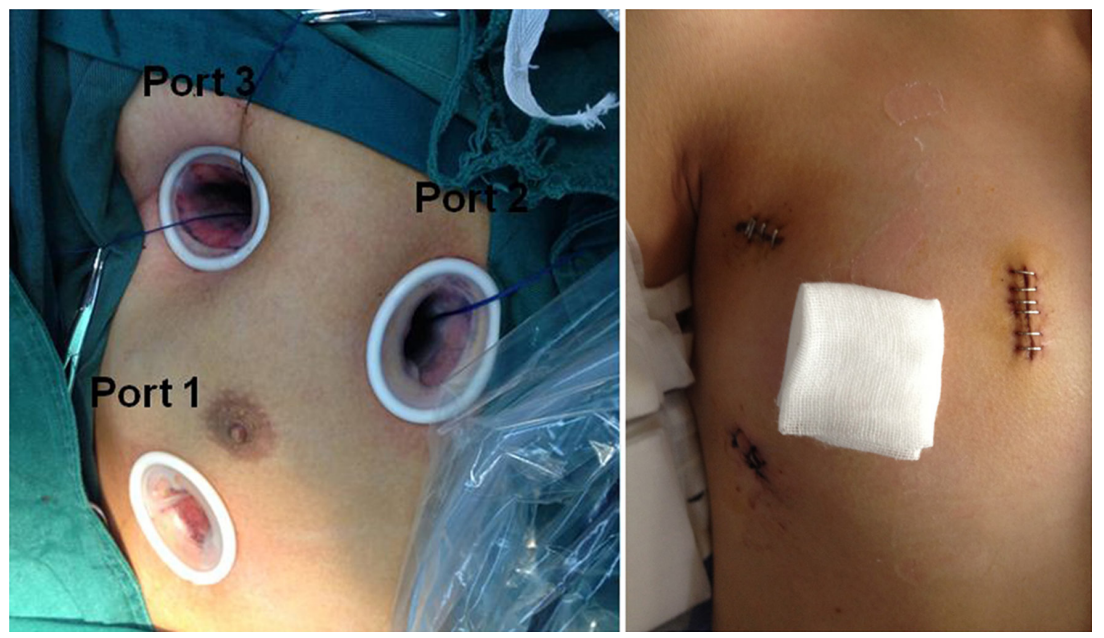

Figure 1 The locations of three chest incisions.

placed through the chest wall.

The inferior and superior vena cava were encircled with linen tapes. Transesophageal echocardiography (TEE) was positioned before heparinization and used to assess the position of the catheter in the superior vena cava. In group B, "Chitwood" cross-clamp (Scanlan International Inc, St. Paul, MN, USA) was used to perform aortic occlusion through Port 3 and antegrade cold HTK cardioplegic solution was directly administered through Port 3 with a $12 \mathrm{~F}$ cannula inserted into the root of the aorta after aortic occlusion, in which de-airing was carried out. Carbon dioxide was continuously injected into the right chest for air displacement in both groups. A surgical field was displayed on a screen with assistance of thoracoscope through Port 1.

In the two groups, the right atriotomy was performed parallel to the atrioventricular groove. In group A, a suction tube was positioned in the coronary sinus ostium area through Port 1 to keep the operation field bloodless. After the right atriotomy, retraction stitch of $2 / 0$ silk was placed across the catheter between the inferior and superior vena cava to prop the wall of the right atrium up to provide a clear surgery field. Simultaneously, catheter position in the superior vena cava was assessed again by TEE. In both groups, ASD closure was performed by direct 4-0 polyester suture, and a Dacron or bovine patch was used to repair the large ASD and the ASD without rim using running polyester suture.

For patients in group A, left atrial de-airing was performed by holding positive pressure on the lungs to expel blood from the left atrium with help of anesthetist at the end of the closure of ASD; conversely, in group B, de-airing was performed by aspirating the air through the perfusion needle in the aortic root. TEE was employed in the procedure to assess the integrity of the closure of ASD. A 24F tube was positioned in the right pleura space through Port 1 for drainage.

\section{Perioperative management}

Before the operation, femoral artery and vein Doppler ultrasound or computed tomography angiography (CTA) examination was used to evaluate diameter of the femoral artery and vein, the presence of plaque and vascular malformations. Two-dimensional (2D) transthoracic echocardiography and color-flow Doppler were used to establish the size, type, and location of ASD connected with possible associated anomalies. Pulmonary function test and arterial blood gas analysis were performed to evaluate the respiratory function. Post-operation care measures, possible complications, and clinical outcomes were provided to all patients and their family members. Following the operation, patients were monitored in the intensive care unit (ICU). All patients received mechanical ventilation with a peak end-expiratory pressure (PEEP) of $5 \mathrm{cmH}_{2} \mathrm{O}$. Bedside chest $\mathrm{X}$-ray and echocardiogram were performed in ICU to assess the heart function and exclude lung complications. Mechanical ventilation was removed once spontaneous respiration and hemodynamics were stabilized.

\section{Statistical analysis}

Statistical analysis was performed using SPSS 21.0 software 
Table 2 Comparison of operational data between two groups

\begin{tabular}{llll}
\hline Variables & $\begin{array}{l}\text { Group A } \\
(\mathrm{n}=115)\end{array}$ & $\begin{array}{l}\text { Group B } \\
(\mathrm{n}=46)\end{array}$ & P value \\
\hline Operation time (min) & & & \\
With Dacron/bovine patch & $169.1 \pm 10.9$ & $197 \pm 11.9$ & $<0.001$ \\
Without Dacron/bovine patch & $159.5 \pm 10.4$ & $183 \pm 11.7$ & $<0.001$ \\
CPB time (min) & & & \\
With Dacron/bovine patch & $60.2 \pm 10.3$ & $78.1 \pm 12.7$ & $<0.001$ \\
Without Dacron/bovine patch & $54.9 \pm 9.1$ & $74.9 \pm 8.7$ & $<0.001$ \\
Aortic cross-clamp time (min) & 0 & $41.2 \pm 5.8$ & 0.000 \\
Mechanical ventilation time (h) & $7.5 \pm 2.7$ & $9.7 \pm 4.9$ & 0.001 \\
ICU stay (h) & $14.9 \pm 3.2$ & $17.3 \pm 7.1$ & 0.003 \\
Postoperative hospital stay (d) & $6.8 \pm 1.4$ & $8.0 \pm 2.6$ & 0.001 \\
Blood transfusion during & $17(14.8 \%)$ & $11(23.9 \%)$ & 0.36 \\
operation & & & \\
Volume of thoracic drainage & $267.5 \pm 76.3$ & $270.1 \pm 68.4$ & 0.98 \\
(mL) & & & \\
\hline
\end{tabular}

ICU, intensive care unit; CPB, cardiopulmonary bypass.

(IBM, Armonk, NY, USA). Quantitative variables were presented as mean \pm standard deviation $(\mathrm{SD})$. The oneway analysis of variance (ANOVA) was used to determine whether there were any statistically significant differences between the means of the two groups. Categorical data were analyzed by the Chi-squared test. $\mathrm{P}$ value $<0.05$ was considered statistically significant.

\section{Results}

Total thoracoscopic ASD closures on both groups were successful without serious complications in all patients, including 48 patients with primary sutures and 113 patients with Dacron or bovine patches. There was no reverting from the thoracoscopic procedure to a sternotomy in both groups. There was also no reoperation for bleeding, femoral vessel injuries, in-hospital mortality, or other cardiovascular complications. As shown in Table 1, there were no statistically significant differences between group A and group B in age, sex, weight, eject fraction, pulmonary artery systolic pressure (PASP) or size of ASD.

As shown in Table 2, duration of operation and CPB time with or without Dacron or bovine patch in group A was shorter than group B. Patients in group A had shorter aorta cross-clamped time, duration of mechanical ventilation, the length of ICU, and duration of post-operative hospital stay than group B. There was no statistically significant difference in blood transfusion during operation or postoperation thoracic drainage. The mean follow-up time of group A and group B was $370 \pm 27$ and $366 \pm 20$ days, respectively. Echocardiograms at 3, 30, 90 and 365 days after operation were assessed at each follow-up visit, and showed no residual shunts or tricuspid regurgitation.

\section{Discussion}

Over the past few years, the significant advantages of minimizing surgical trauma with assistance of endoscopy have resulted in a number of total endoscopic cardiac surgical procedures $(6,11,14)$. Ma et al. reported a singlecenter experience on total endoscopic closure for ASD without assistance of a robotic-surgical system $(7,11,15)$. It has been reported that total endoscopic repair for ASD was associated with a faster recovery of physical function and a superior quality of life compared with conventional sternotomy (15). Xiao et al. reported a series of cases of total thoracoscopic closure of ASD on beating hearts with the da Vinci Surgical System (6). We previously reported that the total thoracoscopic ASD repair resulted in a lower level of systemic inflammatory response (SIRS) after CPB compared with conventional surgery through a sternotomy, including the level of tumor necrosis factor- $\alpha(\mathrm{TNF}-\alpha)$, interleukin-6 (IL-6), interleukin-10 (IL-10), and intercellular adhesion molecule 1 (ICAM-1), and reduced the damage to tissue and organs (9).

In a previous report, stay suture or atrial retractor, which might need more incisions on the chest wall, was placed in the right atrium to expose the ASD. Based on the previous reports (6,10-13), we improved the techniques and skills of the total endoscopic closure for ASD without assistance of the da Vinci Surgical System which made the procedure much more appropriate and feasible. In the present study, a 2-0 silk stitch was placed across the catheter between the inferior and superior vena cava and pulled out of the chest wall through Port 2. The catheter held up the right atrium wall, which played the role of right atrial retractor and had no more incision (Figure 2). However, more attention should be paid to movement of catheter in the superior vena cava, which might negatively affect the drainage of superior vena cava. TEE could help assess position of the catheter as well.

In this study, we reported the maximum number of cases who underwent total endoscopic closure for ASD on beating hearts without assistance of a robotic-surgical system. Injuries 


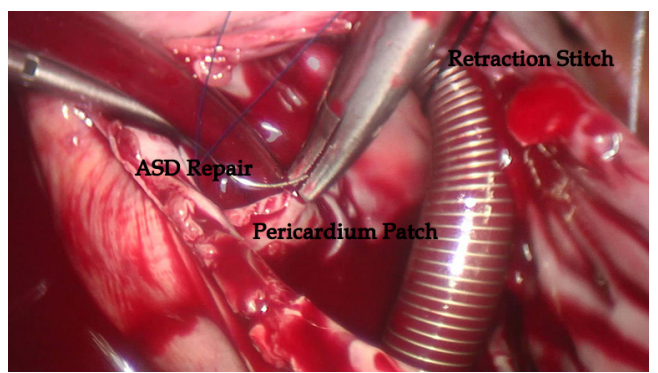

Figure 2 Closure for ASD. ASD, atrial septal defect.

caused by cardiovascular surgery were mainly associated with duration of $\mathrm{CPB}$ and hearts arrested with cardioplegia. It is well-known that CPB makes great contribution to the SIRS syndrome and activates the coagulation system, the fibrinolytic system, the complement system, endothelial cells and platelets. All of the above-mentioned factors might often lead to major organ dysfunction and even death (16). In our study, we found the operation time and $\mathrm{CPB}$ time of group A were both significantly shorter than group B, which may result in less injuries to tissues and organs. Pendse et al. (17) reported a number of patients who underwent ASD repair on beating heart. They found that there was no postoperative death or other serious complication associated with the procedure of ASD repair on beating hearts (17). In addition, it has been demonstrated that hearts arrested with cardioplegia was associated with the pathophysiology of myocardium ischemia-reperfusion injury (17). Proximal aortic arteriosclerosis was the source of macro- and microembolism produced at the time of placement and release of the cross-clamp. Procedures of ASD repair on beating hearts didn't need an aorta cross-clamp and avoided the abovementioned injuries. In the present study, post-operative mechanical ventilation was required in all patients of both groups. The duration of mechanical ventilation of group A was shorter than group B. Shorter duration of mechanical ventilation could decrease the possibilities of pulmonary infection and ventilation associated pneumonia. In group A, patients underwent total endoscopic ASD closure on beating heart without aorta cross-clamped. The advantages of this technique included performance of surgery with the heart in a more physiological state, avoiding of ischaemia-reperfusion injury, and decrease of embolism.

\section{Limitation}

This study had several limitations. First, the study is a single-center study and only a small number of patients were involved. In addition, the anatomic classification of ASD influenced the selection of surgical technique. Another limitation is that the length of stay in the ICU, duration of mechanical ventilation, and duration of post-operative hospital stay can be influenced by some other subjective factors.

In conclusion, we reported that the largest group of patients to date receiving total thoracoscopic ASD repair on beating hearts without assistance of a robotic-surgical system. The procedure is safe and has a shorter operation and $\mathrm{CPB}$, a shorter length of stay in the ICU and duration of post-operative hospital stay.

\section{Acknowledgements}

We thank all the colleagues of our cardiac team for presenting their valuable suggestions.

Funding: This work was supported by Grant of Nanjing City [201715002].

\section{Footnote}

Conflicts of Interest: The authors have no conflicts of interest to declare.

Ethical Statement: This study was approved by the Institutional Review Board of the First Affiliated Hospital with Nanjing Medical University (Nanjing, Jiangsu province, China) (No. 2018-SR-249). Written informed consent was obtained from the patients for publication of their data and any accompanying images.

\section{References}

1. Geva T, Martins JD, Wald RM. Atrial septal defects. Lancet 2014;383:1921-32.

2. Kuijpers JM, Mulder BJ, Bouma BJ. Secundum atrial septal defect in adults: a practical review and recent developments. Neth Heart J 2015;23:205-11.

3. El-Said H, Hegde S, Foerster S, et al. Device therapy for atrial septal defects in a multicenter cohort: acute outcomes and adverse events. Catheter Cardiovasc Interv 2015;85:227-33.

4. Gervasi L, Basu S. Atrial septal defect devices used in the cardiac catheterization laboratory. Prog Cardiovasc Nurs 2009;24:86-9.

5. Baruteau AE, Hascoët $S$, Fraisse A. Transthoracic 
echocardiography is a safe alternative for assessment and guidance of transcatheter closure of secundum atrial septal defect in children. J Thorac Dis 2017;9:1247-56.

6. Xiao C, Gao C, Yang M, et al. Totally robotic atrial septal defect closure: 7-year single-institution experience and follow-up. Interact Cardiovasc Thorac Surg 2014;19:933-7.

7. Ma ZS, Dong MF, Yin QY, et al. Totally thoracoscopic repair of atrial septal defect without robotic assistance: a single-center experience. J Thorac Cardiovasc Surg 2011;141:1380-3.

8. Fortunato Júnior JA, Pereira ML, Martins AL, et al. Video-assisted cardiac surgery: 6 years of experience. Rev Bras Cir Cardiovasc 2012;27:24-37.

9. Liu X, Wu Y, Zhu J, et al. Totally thoracoscopic repair of atrial septal defect reduces systemic inflammatory reaction and myocardial damage in initial patients. Eur J Med Res 2014;19:13.

10. Argenziano M, Oz MC, Kohmoto T, et al. Totally endoscopic atrial septal defect repair with robotic assistance. Circulation 2003;108 Suppl 1:II191-4.

11. Ma ZS, Dong MF, Yin QY, et al. Totally thoracoscopic closure for atrial septal defect on perfused beating hearts.
Eur J Cardiothorac Surg 2012;41:1316-9.

12. Bonaros N, Schachner T, Oehlinger A, et al. Robotically assisted totally endoscopic atrial septal defect repair: insights from operative times, learning curves, and clinical outcome. Ann Thorac Surg 2006;82:687-93.

13. Fortunato JA Jr, Branco Filho AA, Branco A, et al. Standardization of video-assisted cardiac surgery technique: initial experience. Rev Bras Cir Cardiovasc 2008;23:183-9.

14. Thapmongkol S, Sayasathid J, Methrujpanont J, et al. Beating heart as an alternative for closure of secundum atrial septal defect. Asian Cardiovasc Thorac Ann 2012;20:141-5.

15. Ma ZS, Yin QY, Dong MF, et al. Quality of life in patients undergoing totally thoracoscopic closure for atrial septal defect. Ann Thorac Surg 2011;92:2230-4.

16. Day JR, Taylor KM. The systemic inflammatory response syndrome and cardiopulmonary bypass. Int J Surg 2005;3:129-40.

17. Pendse N, Gupta S, Geelani MA, et al. Repair of atrial septal defects on the perfused beating heart. Tex Heart Inst J 2009;36:425-7.

Cite this article as: Tang $\mathrm{Y}, \mathrm{Wu} \mathrm{Y,} \mathrm{Zhu} \mathrm{J,} \mathrm{Liu} \mathrm{X,} \mathrm{Zhou} \mathrm{J,}$ Huang H, Li M, Dai Y, Han X. Total endoscopic repair of atrial septal defect under on-pump beating heart. J Thorac Dis 2018;10(12):6557-6562. doi: 10.21037/jtd.2018.10.89 